\title{
High time resolution observations of millisecond pulsars with the Fermi Large Area Telescope
}

\section{Lucas Guillemot*, on behalf of the Fermi LAT collaboration}

Max-Planck-Institut für Radioastronomie, Auf dem Hügel 69, 53121 Bonn, Germany

E-mail: guillemo@mpifr-bonn.mpg •de

Since Fermi was launched in June 2008, the number of known gamma-ray pulsars has increased dramatically: pulsed gamma-ray emission has been detected from more than 60 pulsars by the Large Area Telescope (LAT), the main instrument aboard the Fermi satellite. Among these detections, a population of gamma-ray millisecond pulsars (MSPs) has been seen for the first time. The timing analysis of these rapidly-rotating (rotational period below $30 \mathrm{~ms}$ ) and relatively faint (a few tens to hundreds of gamma-ray photons per year) objects illustrates the importance of highly-accurate photon time stamps, and the multi-wavelength monitoring of the pulsar rotational period as a function of time. These elements allow the LAT to observe variations in the light curve of MSPs at the $10 \mu \mathrm{s}$ level, for photons accumulated over a year. I review the observations of MSPs with the Fermi LAT, and discuss the recent discovery of numerous MSPs at the position of unassociated Fermi sources with high time resolution radio observations.

High Time Resolution Astrophysics IV - The Era of Extremely Large Telescopes - HTRA-IV,

May 5-7, 2010

Agios Nikolaos, Crete, Greece

\footnotetext{
* Speaker.
} 


\section{Timing observations of pulsars and millisecond pulsars}

High time resolution astronomy generally refers to the study of astrophysical phenomena occurring in time scales of a fraction of a second. Studies of gamma-ray emission from pulsars are currently unfeasible on such short time scales. However, what makes the emission from pulsars specific is precisely the pulsed nature of the emission. One can exploit that periodicity of the signal by integrating gamma-ray photons over a long period of time with accurate time stamps, and perform high resolution variability studies of the intensity across the pulsar's rotation (the so-called light curve). A number of observables are available through these timing observations:

- Is there any pulsed emission coming from a particular position in the sky? Detection (or not) of pulsed gamma-ray emission from pulsars helps understand the population of gammaray pulsars in the Galaxy. Theoretical models of high-energy emission from pulsars predict different numbers of gamma-ray pulsars and different properties (see [11] for a review).

- In case of pulsed detection, how structured is the gamma-ray emission profile? The gammaray light curve shape reflects the geometry of regions in the pulsar magnetosphere where the emission is produced. Theoretical models predict different high-energy emission geometries and therefore different emission profiles, that can be confronted with the observed ones to constrain the actual emission geometry.

- Where is the gamma-ray emission located, compared to lower energy emission? For pulsars emitting at lower energies, contemporaneous multi-wavelength observations yield the relative phasing of emission components across the spectrum, and therefore a more complete view of the phenomena occurring in the magnetosphere. Besides, multi-wavelength observations with accurate relative phasing help constrain the ratio of radio-loud to radio-quiet pulsars, which also varies in the different theoretical models, leading to different predictions for the number of observable pulsars in the Galaxy.

These observables require different precision levels for the measurement of rotational phases for individual photons. For detection purposes, rough knowledge of rotational phase as a function of time (for example, accuracy of 5\% of the rotational period), is sufficient. On the other hand, detailed multi-wavelength observations require accurate time-stamping, precise knowledge of the pulsar's rotational behavior over the observations and of dispersive effects that cause low frequency waves to arrive at Earth later than high frequency radiation emitted at the same time. In addition, most millisecond pulsars (MSPs), pulsars with the shortest rotational periods (typically, $\mathrm{P}<30$ $\mathrm{ms}$ ), are in binary orbits which perturbs the apparent rotational period and therefore has to be known accurately. Finally, first year observations of MSPs with the Large Area Telescope (LAT) aboard Fermi [8] showed that gamma-ray MSPs typically emit on the order of 100 to 1000 photons per year $[1,2]$, so that these parameters must be known with great precision over a year or more for future detections of gamma-ray MSPs with the LAT.

\section{Time-stamping of Fermi photons}

As discussed above, accurate time-stamping of high-energy photons recorded by the Fermi LAT is crucial for the study of pulsars in general and millisecond pulsars in particular. The pre- 
launch requirement for the timing precision of individual gamma-ray photons was of $10 \mu \mathrm{s}$, sufficient for detailed temporal analysis of the fastest pulsars. Previous high-energy satellite experiments such as USA, Chandra, XMM or CGRO have however experienced problems with photon time-stamping ${ }^{1}$. These issues were diagnosed after launch, making them difficult to solve, as dates then have to be calibrated using reference astrophysical objects, and their inherent timing stability limiting the accuracy of the calibration.

For these reasons, the timing accuracy of the LAT has been checked before launch, by comparing times recorded by the LAT and a muon telescope placed next to Fermi, for atmospheric muons detected by both instruments ${ }^{2}$. The muon telescope, consisting of scintillators and photomultiplier tubes, was coupled to a reference GPS receiver measuring the time stamps, whose accuracy had been demonstrated through optical observations of the Crab pulsar with the CELESTE experiment [12]. The LAT track reconstruction algorithm identified muon events also traversing the external muon telescope. Differences between trigger times for events recorded by the two instruments are shown in Figure 1. A sawtooth pattern, with an amplitude of $1 \mathrm{~ms}$ and a slope of $3.4 \mu \mathrm{s} / \mathrm{s}$ was observed in the first comparison runs, pointing to issues with LAT event dates.

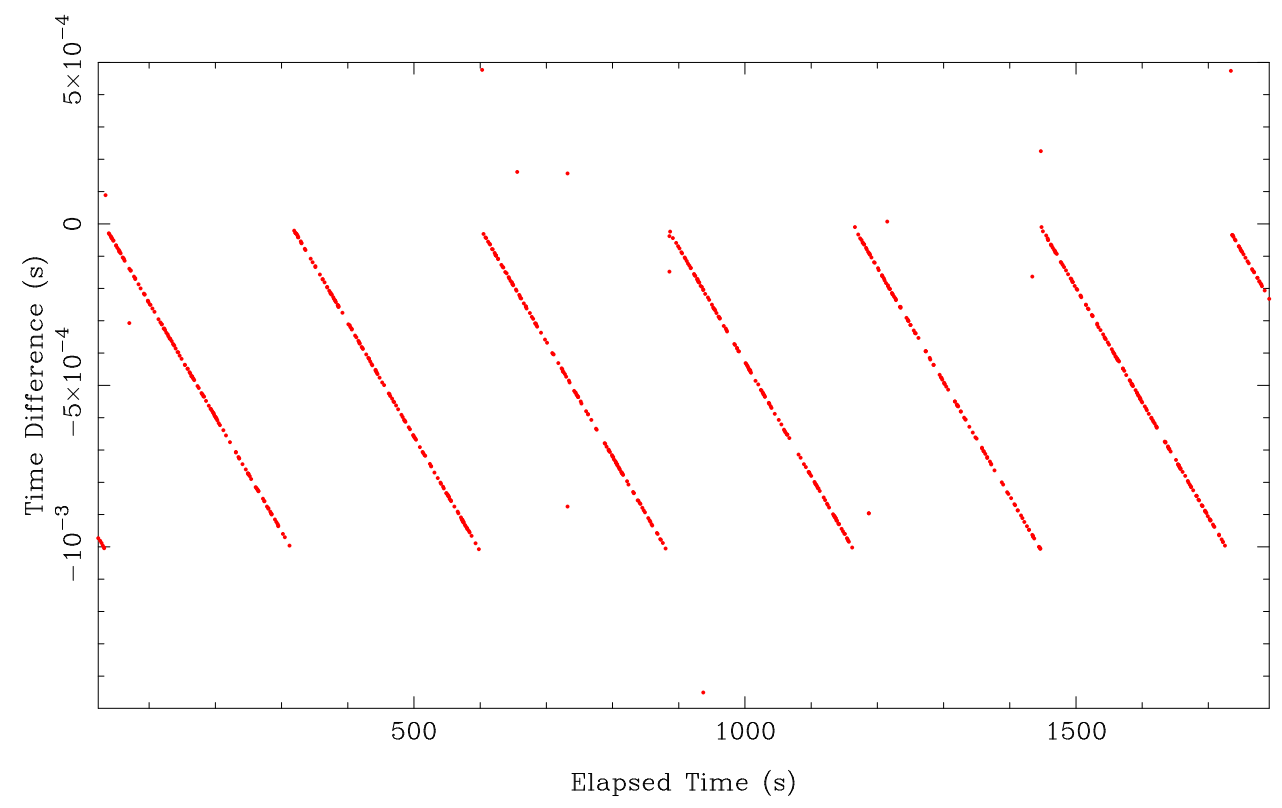

Figure 1: Time difference versus elapsed time for atmospheric muons detected by the Large Area Telescope (LAT) and a muon telescope equipped with a reference GPS, before correction of the Fermi time-stamping software.

${ }^{1}$ For detailed descriptions of these problems, see:

- XMM-Newton: http://xmm.vilspa.esa.es/docs/documents/CAL-TN-0045-1-0.pdf

- Chandra: S. Murray, et al., ApJ 566 (1039), 2002

- ROSAT: http://www.mporzio.astro.it/ gianluca/phdthesis/node28.html

- Details on issues for other missions are available upon request.

${ }^{2}$ These tests were conceived and conducted by D. A. Smith, J. E. Grove, D. P. Sandora and E. J. Siskind. 
A minor error was found in the Fermi spacecraft software interpreting GPS signals and calculating time stamps. This software error was eventually solved, and subsequent tests of the timing accuracy of the LAT revealed agreement between LAT time stamps and those recorded by the muon telescope, to within $1 \mu \mathrm{s}$, as can be seen in Figure 2. For the reasons mentioned above, on-orbit diagnosis would have probably been tedious and would have certainly hindered Fermi LAT studies of millisecond pulsars. This illustrates the importance of pre-launch tests of the timing precision for pulsar science.
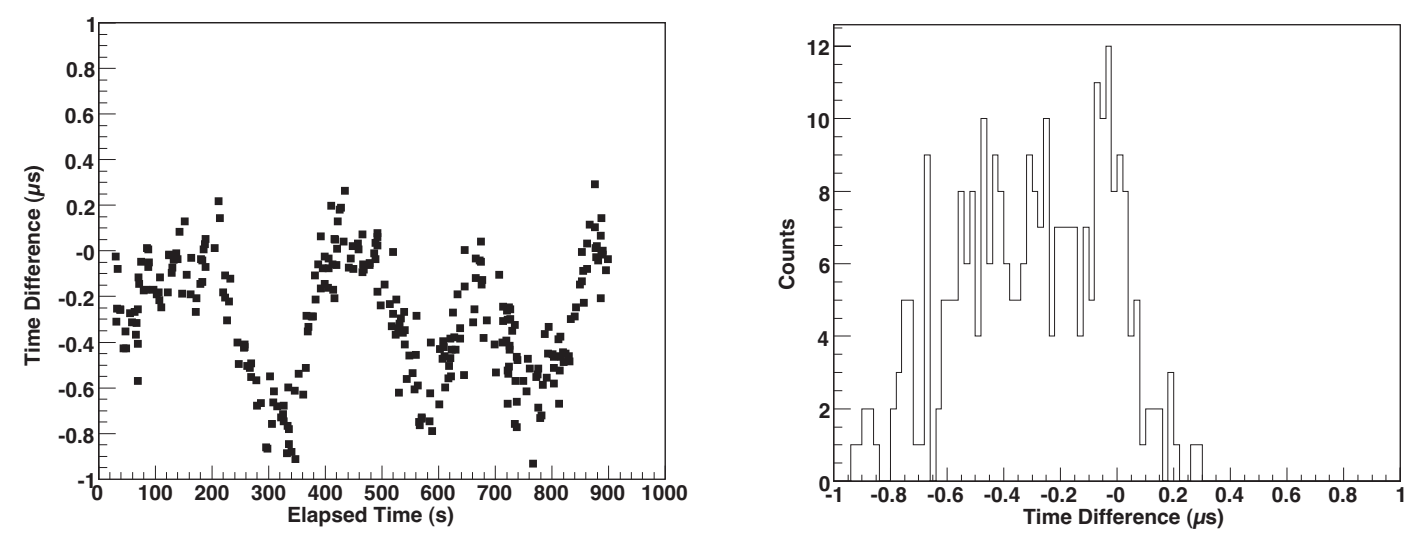

Figure 2: Time difference versus elapsed time and histogram of time differences for atmospheric muons detected by the Large Area Telescope (LAT) and a muon telescope equipped with a reference GPS, after correction of the Fermi time-stamping software.

\section{Multi-wavelength timing observations}

In addition to accurate photon time stamps, the apparent pulsar period as a function of time has to be known precisely to achieve highly-resolved gamma-ray light curves. As mentioned earlier, MSPs are intrinsically faint gamma-ray sources and several months to years of Fermi LAT data are required to detect them. In one year, a $P=5 \mathrm{~ms}$ pulsar rotates more than $6.3 \times 10^{9}$ times, and the apparent rotational period varies with time because of the Earth's motion in the Solar System, the orbital and proper motion of the pulsar if there are any, and the intrinsic spin-down of the pulsar. Therefore, a timing model that accounts for every rotation of the pulsar over several months to years, taking into account the above-mentioned effects, is necessary to record high precision gamma-ray light curves.

To monitor the rotational period of MSPs as a function of time, a pulsar timing campaign involving large radio telescopes around the world as well as X-ray telescopes has been set up before Fermi was launched [15]. For a given pulsar, timing observations are combined to build "ephemerides", which give the astrometric parameters (pulsar position in the sky and proper motion), rotational period and derivatives, binary parameters if any orbital motion, and the dispersion measure (DM), density of free electrons along the line of sight, crucial for the relative phasing of low-frequency light curves with gamma-ray data. Over two hundred pulsars considered best candidates for detection by the Fermi LAT were monitored in this campaign. These pulsars were chosen 
because of their high spin-down luminosity $\dot{E}$, corresponding to the energy loss rate via electromagnetic spin-down, greater than $10^{34} \mathrm{erg} / \mathrm{s}$. More pulsars were later monitored on a regular basis for Fermi, after pulsars below the $10^{34} \mathrm{erg} / \mathrm{s}$ threshold were detected early in the mission (see e.g. [1]). In total, ephemerides have been collected for more than 700 pulsars, reducing the possible bias of gamma-ray pulsation searches with the LAT [4]. In particular, nearly all known Galactic MSPs could be searched for gamma-ray pulsations with accurate timing solutions, as discussed in [2]. Results of this search for gamma-ray pulsations from Galactic field MSPs are discussed below.

\section{Fermi studies of MSPs}

The search for pulsed gamma-ray emission from MSPs has been very successful since Fermi was launched in June 2008. A search for gamma-ray pulsations from the $\simeq 70$ known Galactic field MSPs (i.e., MSPs outside of globular clusters) with ephemerides measured in the timing campaign has led to the detection of pulsations from eight MSPs within nine months of data [1,2]. Since then, high-energy pulsations have been detected for three other MSPs, PSRs J0034-0534, J1939+2134 and J1959+2048 [5, 7] with increased gamma-ray data. The LAT has established MSPs as a prominent class of Galactic gamma-ray sources, and this newly revealed population of gamma-ray MSPs will continue to grow as data accumulate.

Gamma-ray light curves observed for the first eight MSPs detected by the LAT were reminiscent of those of normal gamma-ray pulsars, in terms of peak multiplicity, peak separation and phase shift between radio and gamma-ray emission (see Figure 1 of [2]). The photon statistics were limited though, and complexity might appear with additional data. Nevertheless, the similarity of light curves between the two pulsar populations as well as the similarity of spectral properties (spectral index and cutoff energy) led to the conclusion that the same mechanisms are operating for both pulsar classes [2]. Theoretical modeling of the observed gamma-ray light curves of MSPs confirmed that emission scenarios placing the high-energy in the outer magnetosphere are preferred, similarly to normal pulsars [16]. These fits provided constraints on the pulsar geometry (magnetic axis and line-of-sight inclination angles relative to the rotation axis), generally in agreement with independent measurements.

The three latest gamma-ray MSP detections, PSRs J0034-0534, J1939+2134 and J1959+2048 [5, 7], differ from the first eight MSPs in that their gamma-ray peaks are aligned in phase with radio peaks, as illustrated in Figure 3. Prior to these detections, only one such pulsar was known: the Crab pulsar, for which nearly-aligned emission peaks are observed across the spectrum [3]. For this subclass of pulsars exhibiting alignment of radio and gamma-ray peaks, it has been proposed that the radio and the gamma-ray emission result from caustic formation taking place in co-located regions of the magnetosphere [5].

Figure 4 shows the "spin-down flux" $\dot{E} / D^{2}$, a measure of the rate of kinetic energy loss normalized by the distance squared, as a function of rotational period, for pulsars with known distances. Pulsars detected in gamma rays are those with the highest spin-down flux value, as was also the case for EGRET pulsars. We also note that nearly all MSPs above a threshold of approximately $10^{34} \mathrm{erg} / \mathrm{kpc}^{2}$ have been detected with the Fermi LAT. A good fraction of high $\dot{E} / D^{2}$ normal pulsars have been detected as well. There are several possible reasons for the non-detection of high $\dot{E} / D^{2}$ pulsars. For instance, low Galactic latitude pulsars suffer contamination from the intense Galactic 


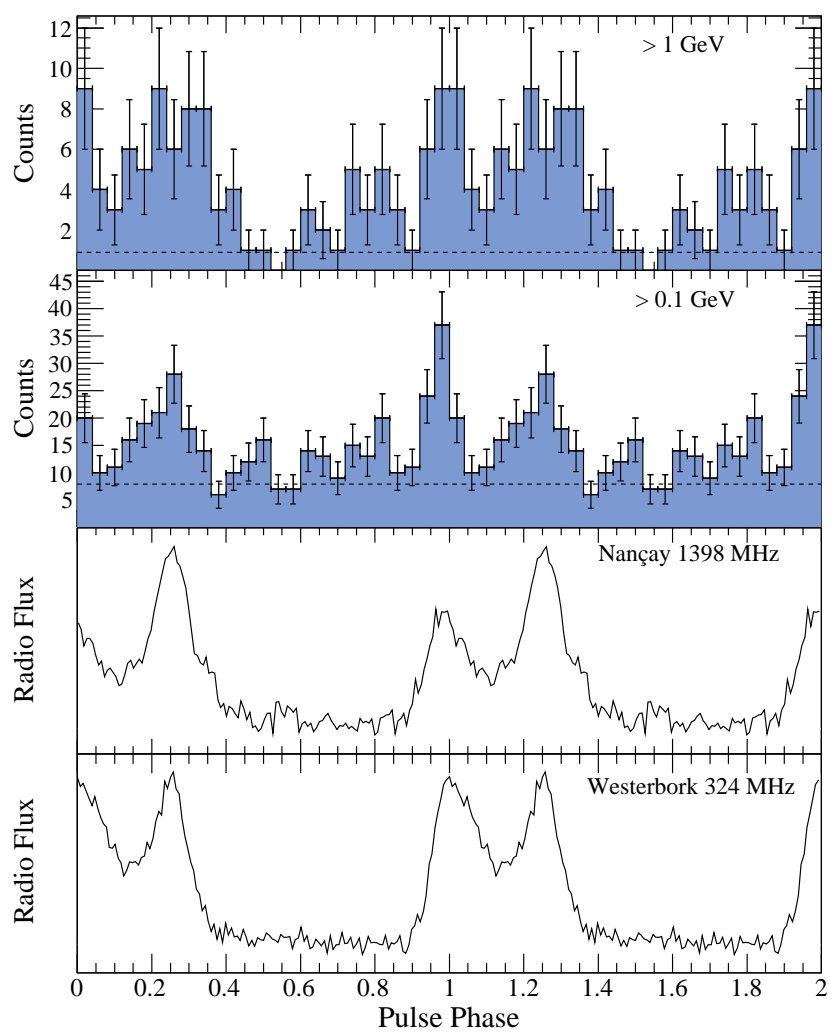

Figure 3: Multi-frequency radio and gamma-ray light curves of PSR J0034-0534 recorded with the Westerbork Synthesis Radio Telescope (WSRT) at $324 \mathrm{MHz}$, the Nançay radio telescope at $1398 \mathrm{MHz}$ and the LAT above $0.1 \mathrm{GeV}$ and above $1 \mathrm{GeV}$ within $0.8^{\circ}$ of the pulsar's position. Two pulsar rotations are shown for clarity. Figure adapted from [5].

diffuse background, making them difficult to detect. Distances are generally uncertain and some high $\dot{E} / D^{2}$ may be more distant, thereby reducing their spin-down flux. Some high $\dot{E} / D^{2}$ pulsars may also be bright gamma-ray emitters, but with gamma-ray beams that do not point towards the Earth, or may also not emit gamma rays at all. Detailed studies of the geometrical orientation of individual objects (angle between the magnetic axis and the rotation axis, $\alpha$, and angle between the line-of-sight and the rotation axis, $\zeta$ ) might help understand causes of non-detection.

Interestingly, the gamma-ray detectability of pulsars does not seem to be linked to the intensity of the magnetic field at the stellar surface, but is clearly correlated with the magnetic field strength at the light cylinder, $B_{L C} \propto \sqrt{\dot{E} / P^{2}}$, as shown in Figure 5. Normal pulsars and MSPs detected with the LAT lie in the upper part of the plot, confirming that gamma-ray emission from pulsars seems to originate at high altitude in the magnetosphere.

\section{New MSPs found in Fermi sources}

In the previous section we described the search for gamma-ray pulsations from already known Galactic MSPs, folded at their rotational period. All millisecond pulsars in this sample have been discovered in radio surveys, so that gamma-ray observations of MSPs were so far limited by past 


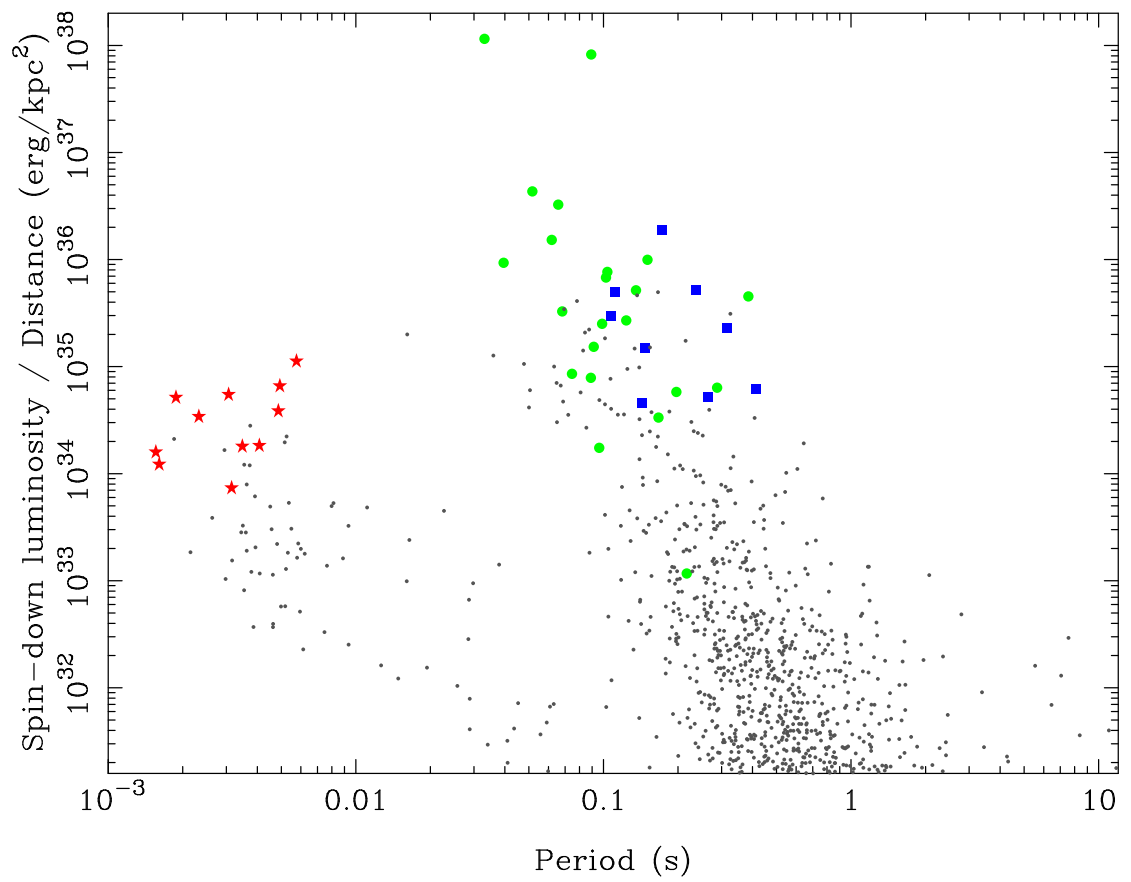

Figure 4: "Spin-down flux" $\dot{E} / D^{2}$ as a function of rotational period for Galactic field pulsars with known distances. Red triangles indicate gamma-ray millisecond pulsars, blue squares show the LAT-discovered pulsars (accurate distances are unavailable for most of these pulsars, so this plot only shows a subset of LAT-discovered pulsars) and green circles are normal radio-emitting objects. Gray dots are pulsars currently undetected in gamma rays.

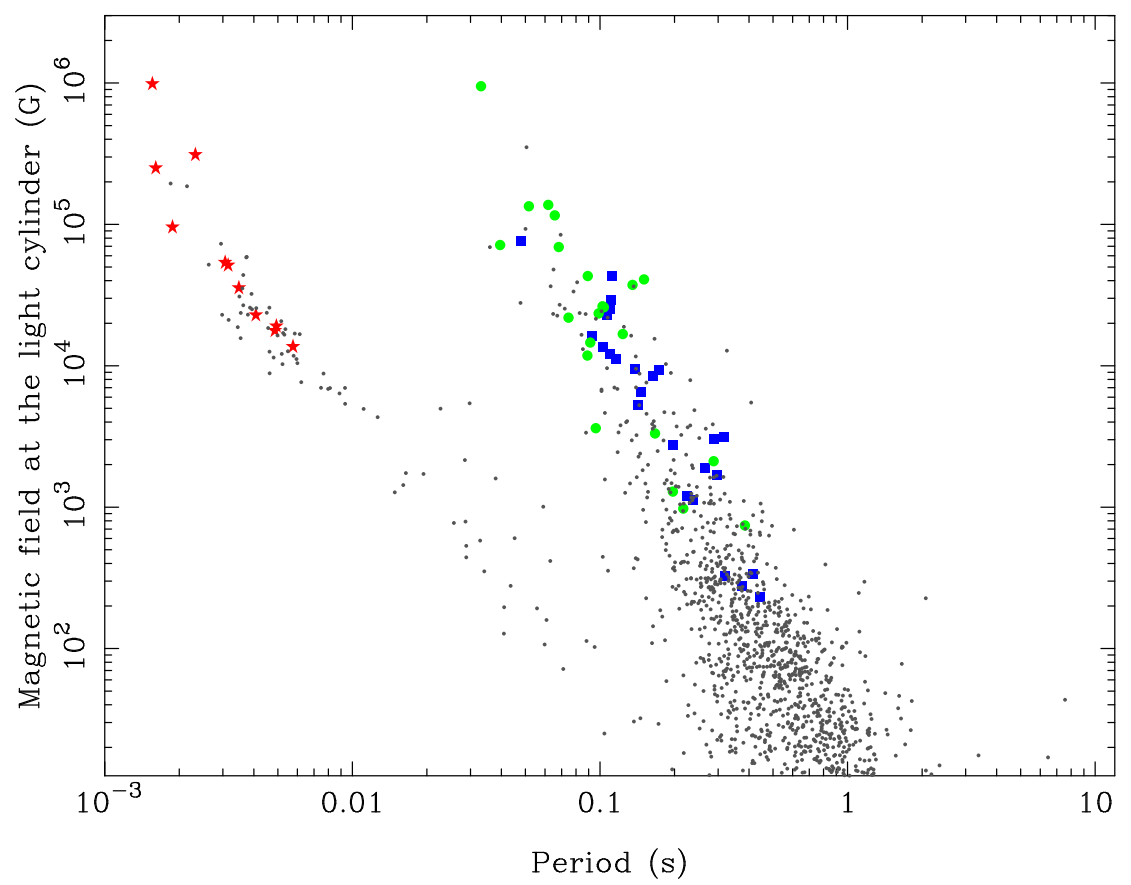

Figure 5: Magnetic field strength at the light cylinder $B_{L C}$ as a function of rotational period for known pulsars in the Galactic field. 
radio searches for MSPs, which have covered the sky inhomogeneously. The detection of gammaray MSPs in good number established these objects as an important class of gamma-ray sources. Therefore, some of the Fermi LAT sources with no known counterparts and with spectral properties similar to those of known gamma-ray MSPs (spectra with sharp cutoffs at a few $\mathrm{GeV}$ and no flux variability with time) must be currently unknown MSPs. Because the gamma-ray data are very sparse and most MSPs are in binary orbits, direct searches for gamma-ray pulsations with Fourier techniques are challenging. However, assuming that these MSPs emit radio pulses crossing the Earth's line-of-sight, one can search for periodicity by doing high time resolution observations of the LAT sources in the radio domain, where pulsars are detected in time scales of less than a minute to a few hours.

Over the last few months a search for radio pulsations at the position of LAT unassociated sources, such as those listed in the Fermi Large Area Telescope First Source Catalog [6], has been conducted at different radio telescopes around the world, resulting in the discovery of 18 new MSPs at the Green Bank, Parkes, Nançay, and Effelsberg telescopes. As can be seen in Figure 6, which shows the location of the 18 new MSPs as well as the 11 already known MSPs detected in gamma rays using pulsar ephemerides, MSPs have mostly been discovered at high Galactic latitudes. Searches are ongoing, therefore other MSPs should be discovered in the future. However, these 18 MSPs discovered in a few months already represent a 25\% increase of the population of known MSPs in the Galactic field, the first MSP having been found thirty years ago [9].

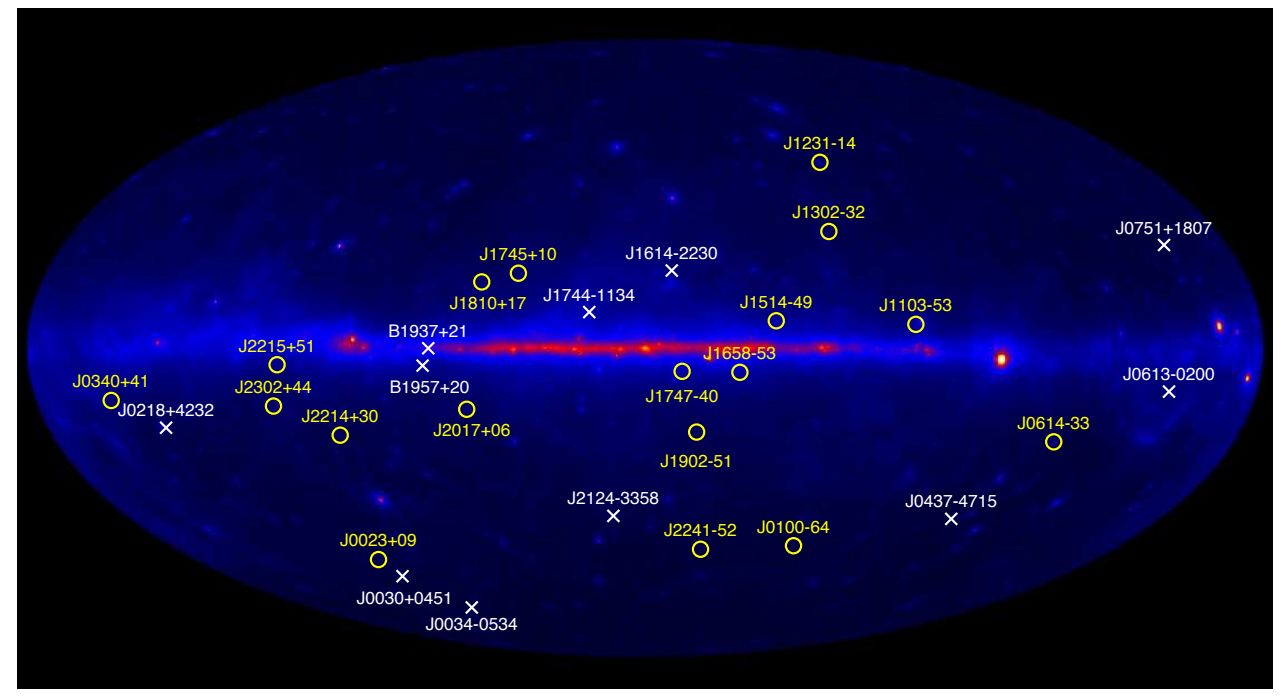

Figure 6: Sky map of the data taken by the Fermi LAT between 4 August 2008 and 2 December 2010, in Galactic coordinates. White crosses indicate the 11 MSPs for which gamma-ray pulsations have been observed, using radio ephemerides. Yellow circles show the 18 radio MSPs discovered at the position of LAT unassociated sources, as of June 2010. Figure adapted from [14]. Credit: Aous Abdo, Paul Ray \& Pablo Saz Parkinson.

These discoveries open a number of prospects for pulsar science. First, as the MSPs have been found at the position of Fermi LAT unassociated sources, it is reasonable to think that they power the gamma-ray source, and therefore gamma-ray pulsations should be observable when the radio ephemeris is accurate enough for folding the gamma-ray data recorded by the LAT. Five of 
them have already been detected as pulsed sources of gamma rays. [10, 13]. Assuming all 18 new MSPs are gamma-ray emitters, the number of gamma-ray MSPs known currently becomes 29, an important fraction of all gamma-ray pulsars. Also, five of the 18 new MSPs are in "Black Widow" type binary systems, orbiting light white dwarf companion stars, which doubles the known sample of such binary systems. Finally, some of the new MSPs with bright radio emission and sharp features in their light curves may be interesting for $\mathrm{nHz}$ gravitational wave detection projects using pulsar timing, such as $\mathrm{LEAP}^{3}$ or NANOGrav ${ }^{4}$.

\section{Conclusion}

Pulsed gamma-ray emission has been observed for a total of 16 MSPs so far with the LAT aboard Fermi, and this number is expected to grow as data accumulate and previously unknown gamma-ray MSPs are discovered in LAT unassociated sources. MSPs therefore represent an important fraction of gamma-ray pulsars known to date. The unprecedented sensitivity of the LAT, its accurate time stamps corrected and validated before launch, and the multi-wavelength timing observations in support of Fermi providing contemporaneous ephemerides for pulsars were key to these numerous detections which help us understand the Galactic population of gamma-ray sources. Even though the data are sparse, the gamma rays can be folded over time scales of months to years to obtain highly-detailed views of the high-energy emission regions in the magnetosphere of pulsars. These observations are valuable inputs for theoretical models of high-energy emission from pulsars as they allow to confront their predictions with actual light curves, thereby improving our comprehension of physical mechanisms occurring in the magnetosphere of these objects. In addition to helping understand the physics of gamma-ray emission from pulsars, the Fermi LAT also helps discover normal and millisecond pulsars at lower frequencies, including pulsars in black widow systems with interesting formation scenarios and pulsars with very stable rotation, candidate targets for pulsar timing array projects. Fermi is therefore playing a key role in pulsar astronomy, and there are all reasons to believe that it will continue to do so in the next few years.

\section{Acknowledgements}

The Fermi LAT Collaboration acknowledges generous ongoing support from a number of agencies and institutes that have supported both the development and the operation of the LAT as well as scientific data analysis. These include the National Aeronautics and Space Administration and the Department of Energy in the United States, the Commissariat à l'Énergie Atomique and the Centre National de la Recherche Scientifique / Institut National de Physique Nucléaire et de Physique des Particules in France, the Agenzia Spaziale Italiana and the Istituto Nazionale di Fisica Nucleare in Italy, the Ministry of Education, Culture, Sports, Science and Technology (MEXT), High Energy Accelerator Research Organization (KEK) and Japan Aerospace Exploration Agency (JAXA) in Japan, and the K. A. Wallenberg Foundation, the Swedish Research Council and the Swedish National Space Board in Sweden.

\footnotetext{
${ }^{3}$ http://epta.jb.man.ac.uk/leap.html

${ }^{4}$ http://nanograv.org/
} 
Additional support for science analysis during the operations phase is gratefully acknowledged from the Istituto Nazionale di Astrofisica in Italy and the Centre National d'Études Spatiales in France.

\section{References}

[1] A. A., Abdo, et al., Pulsed Gamma Rays from the Millisecond Pulsar J0030+0451 with the Fermi Large Area Telescope, ApJ 699 (1171), 2009

[2] A. A., Abdo, et al., A Population of Gamma-Ray Millisecond Pulsars Seen with the Fermi Large Area Telescope, Science 325 (848), 2009

[3] A. A., Abdo, et al., Fermi Large Area Telescope Observations of the Crab Pulsar And Nebula, ApJ 708 (1254), 2010

[4] A. A., Abdo, et al., The First Fermi Large Area Telescope Catalog of Gamma-ray Pulsars, ApJS 187 (460), 2010

[5] A. A., Abdo, et al., Discovery of Pulsed $\gamma$-Rays from PSR J0034-0534 with the Fermi Large Area Telescope: A Case for Co-Located Radio and $\gamma$-Ray Emission Regions, ApJ 712 (957), 2010

[6] A. A., Abdo, et al., Fermi Large Area Telescope First Source Catalog, ApJS 188 (405), 2010

[7] A. A., Abdo, et al., in preparation, 2010

[8] W. B., Atwood, et al., The Large Area Telescope on the Fermi Gamma-Ray Space Telescope Mission, ApJ 697 (1071), 2009

[9] D. C., Backer, S. R. Kulkarni, C. Heiles, M. M. Davis, \& W. M. Goss, A millisecond pulsar, Nature $300(615), 1982$

[10] I., Cognard, et al., in preparation, 2010

[11] A. K., Harding, I. A., Grenier, \& P. L. Gonthier, The Geminga fraction, Ap\&SS 309 (221), 2007

[12] M., de Naurois, et al., Measurement of the Crab Flux above $60 \mathrm{GeV}$ with the CELESTE Cerenkov Telescope, ApJ 566 (343), 2002

[13] S. M., Ransom, et al., New Radio Millisecond Pulsars in Fermi (Formerly) Unassociated Sources, HEAD 41 (655), 2010

[14] P. S., Ray, P. M. Saz Parkinson, et al., Pulsar Results with the Fermi Large Area Telescope, proceedings of the "High-energy emission from pulsars and their systems" workshop in Sant Cugat, 2010

[15] D. A., Smith, L., Guillemot, F., Camilo, et al., Pulsar timing for the Fermi gamma-ray space telescope, A\&A 492 (923), 2008

[16] C., Venter, A. K., Harding, \& L., Guillemot, Probing Millisecond Pulsar Emission Geometry Using Light Curves from the Fermi/Large Area Telescope, ApJ 707 (800), 2009 